\title{
SEGREGAÇÃO DE RESÍDUOS NOS SERVIÇOS DE SAÚDE: A EDUCAÇÃO AMBIENTAL EM UM HOSPITAL-ESCOLA*
}

Laura Christina Macedo ${ }^{1}$, Liliana Muller Larocca ${ }^{1}$, Maria Marta Nolasco Chaves ${ }^{1}$, Paulo Oliveira Perna ${ }^{1}$, Sandra M. A.Muntsch ${ }^{1}$, Emanuelle F. Castilho Damaceno ${ }^{2}$, Thaís Sanglard de Souza ${ }^{2}$, Carolina Bolfe Poliquesi², Thiago C. Truppel ${ }^{2}$, Claudia de Souza ${ }^{2}$

RESUMO: O gerenciamento dos resíduos dos serviços de saúde desperta interesse dos profissionais da área devido às repercussões que ocasionam no meio ambiente, na saúde dos trabalhadores e da população. Isso levou à elaboração de legislações que dispõem sobre o tema e regulamentam a implantação de programas que visam o correto manejo de resíduos. No entanto, somente essas leis não garantem que os profissionais e as instituições realizem o gerenciamento dos resíduos, sendo necessário o desenvolvimento de educação em serviço para resaltar a relevância do assunto. O presente estudo teve como objetivo avaliar o impacto do treinamento em serviço no manejo dos resíduos de saúde. Para tal, comparou-se uma amostra de 15 funcionários treinados com outra de 15 não treinados. Os resultados demonstram que não houve diferença entre os grupos, devido a diversos fatores. Apesar disso, acredita-se que a educação em serviço é de extrema relevância por mediar a transformação dos sujeitos.

PALAVRAS-CHAVE: Resíduos de Serviços de Saúde; Capacitação em serviço; Saúde Ambiental

\section{WASTE MANAGEMENT IN HEALTH INSTITUTIONS : ENVIRONMENTAL EDUCATION AT A TEACHING HOSPITAL}

\begin{abstract}
Waste management in health institutions raises the interest of professionals in the area because of the repercussions that they cause in the environment and population's as well as workers' health. This fact led to the elaboration of laws which regulate the issue and the implementation of programs aiming at the correct waste handling. However, only these laws are not enough for institutions or professionals to carry out waste management. It is necessary the development of professionals' education so that they realize the relevance of the subject. The study had as general objective to evaluate the impact of the in-service training in handling health waste. Thus, trained and not trained caregivers were compared. The results showed that there weren't strong differences between the groups, due to several factors. In spite of that, it is believed that in-service education is extremely important to change citizens' behavior.
\end{abstract}

KEYWORDS: Hospital Waste; Inservice Training; Environmental Health.

\section{SEGREGACIÓN DE LOS RESIDUOS EN LOS SERVICIOS DE SALUD: LA EDUCACIÓN AMBIENTAL EN EL AMBIENTE HOSPITALAR}

RESUMEN: La segregación de los residuos de los servicios de salud despertó interés de los trabajadores del área por causa de las repercusiones que ellos causan en el medio ambiente, en la salud de los trabajadores y de la población. Por eso fueron elaboradas legislaciones que hablan a respecto del tema y reglan la implantación de los programas que tienen como finalidad el correcto manejo. Sin embargo, solamente esas leyes no garantizan que los profesionales y las instituciones ejecuten la segregación de los residuos, por eso es indispensable el desarrollo de la educación en servicio a fin de suscitar la importancia del tema. El objetivo general del estudio fue estimar el impacto de la capacitación en servicio en el manejo de los residuos de salud. Para eso, fue hecha una comparación con una muestra de funcionarios capacitados y no capacitados. Los resultados indican que no hubo diferencias entre los grupos. Con todo, la educación en servicio es muy importante por conducir la transformación en los sujetos.

PALABRAS CLAVE: Residuos de Hospitales; Capacitación en servicio; Salud ambiental.

*Este artigo é parte do trabalho desenvolvido por docentes e alunos do Projeto Licenciar em parceria com a Comissão de Gerenciamento de Resíduos do Hospital de Clínicas da Universidade Federal do Paraná-UFPR.

${ }^{1}$ Enfermeiro. Mestre em Enfermagem. Docente do Departamento de Enfermagem da UFPR.

${ }^{2}$ Aluno de Graduação em Enfermagem integrante do Projeto Licenciar da UFPR.

Autor correspondente:

Laura Christina Macedo

Rua Padre Camargo, 120 - 80060-240 - Curitiba-PR Recebido em: 11/12/06

Email: laurachristina@ufpr.br 


\section{INTRODUÇ̃̃O}

A preocupação com a segregação de Resíduos de Serviços de Saúde (RSS) é algo recente dentro das instituições hospitalares e somente passou a ganhar devida importância na última década, com a aplicação de legislações específicas. As resoluções da Agência Nacional de Vigilância Sanitária (ANVISA) ${ }^{(1)}$ e do Conselho Nacional do Meio Ambiente ${ }^{(2)}$ dispõem sobre o regulamento técnico para o gerenciamento de resíduos de serviços de saúde e tornam obrigatória a qualificação dos profissionais que atuam com tais resíduos. Sobre essa questão,

vários são os fatores que vêm contribuindo para o aumento da geração de resíduos de serviços de saúde nos países desenvolvidos, como o contínuo incremento da complexidade da atenção médica, o uso crescente de material descartável, além do aumento da população idosa que normalmente necessita de mais serviços de saúde e é usuária com mais freqüência de diversos tipos e níveis de especialidades ${ }^{(3: 7)}$

Ressalta-se que para uma melhor caracterização dos resíduos esta qualificação deve ser feita em cada instituição, especificamente, pois existem alguns fatores que interferem na quantidade de resíduos produzidos como

tipo e tamanho do estabelecimento de saúde; quantidade de serviços oferecidos; quantidade de pacientes atendidos; procedimentos médico-hospitalares adotados etc. Normalmente, adota-se uma relação entre a quantidade média dos resíduos de serviços de saúde gerada por dia no estabelecimento com o número de leitos ocupados, resultando em um dado número que poderá servir como um parâmetro comparativo ${ }^{(7: 1984)}$.

O aumento da produção de resíduos nos serviços de saúde tem se constituído em uma preocupação nos hospitais brasileiros. Dados indicam que são coletadas diariamente 228.413 toneladas de resíduos no Brasil. Em geral, estima-se que 1\% desse total corresponda aos resíduos de serviços de saúde, aproximadamente 2.300 toneladas diárias. Os resíduos produzidos pelos serviços de saúde, em $74 \%$ dos municípios brasileiros são depositados a céu aberto, em $57 \%$ são separados nos hospitais e somente em $14 \%$ das cidades brasileiras esses dejetos são tratados adequadamente, conforme previsto pela Legislação vigente $^{(4)}$.

O gerenciamento dos resíduos de saúde tem como objetivo minimizar a produção dos mesmos e proporcionar um encaminhamento seguro, visando a proteção dos trabalhadores e a preservação do ambiente. No que se refere às condições de trabalho, a estrutura e a organização estão relacionadas à sua divisão técnica, ao processo e ao ritmo de trabalho, à distribuição de atividades entre os profissionais, aos níveis de formação e especialização do trabalho. Dependendo da forma como a instituição está estruturada e organizada internamente, os profissionais terão melhores ou piores condições de trabalho ${ }^{(5)}$. Sendo assim, a forma como a instituição lida com a segregação de resíduos passa a ser de extrema importância para a saúde do trabalhador, esteja ele ligado diretamente à assistência ou não.

Em relação à diminuição de custos, a redução de desperdícios de produtos está intimamente ligada ao treinamento e conscientização dos técnicos quanto à influência do modo como realiza os procedimentos na geração de efluentes e resíduos sólidos. Assim, a capacitação dos profissionais é essencial ${ }^{(3)}$.

$\mathrm{O}$ gerenciamento de resíduos, segundo a ANVISA $^{(1)}$ é composto pelas etapas: segregação, que consiste na separação dos resíduos no momento e local de sua geração, de acordo com as características físicas, químicas, biológicas, o seu estado físico e os riscos envolvidos; acondicionamento, que se trata do ato de embalar os resíduos segregados em sacos ou recipientes que evitem vazamentos e resistam às ações de punctura e ruptura; identificação, que se refere ao conjunto de medidas que permite o reconhecimento dos resíduos contidos nos sacos e recipientes fornecendo informações ao correto manejo dos RSS; transporte interno, que consiste no translado dos resíduos dos pontos de geração até o local destinado ao armazenamento temporário ou armazenamento externo, com a finalidade de apresentação para a coleta.

Ainda compõe o gerenciamento, de acordo com a ANVISA, o armazenamento temporário, que consiste na guarda temporária dos recipientes contendo os resíduos já acondicionados em local próximo aos pontos de geração, visando agilizar a coleta dentro do estabelecimento e otimizar o deslocamento entre os pontos geradores e o ponto destinado à apresentação para coleta externa; $o$ tratamento, que se refere à aplicação de método, técnica ou processo que modifique as características dos riscos inerentes aos resíduos, reduzindo ou eliminando o risco de contaminação, de acidentes ocupacionais ou de dano ao meio ambiente; $o$ armazenamento externo, que consiste na guarda dos 
recipientes de resíduos até a realização da etapa de coleta externa, em ambiente exclusivo, com acesso facilitado para os veículos coletores; a coleta $e$ transportes externos, que consistem na remoção dos RSS do abrigo de resíduos (armazenamento externo) até a unidade de tratamento ou disposição final, utilizando-se técnicas que garantam a preservação das condições de acondicionamento e a integridade dos trabalhadores, da população e do meio ambiente, devendo estar de acordo com as orientações dos órgãos de limpeza urbana; e, por último, a disposição final, que se trata da disposição de resíduos no solo previamente preparado para recebê-los, obedecendo a critérios técnicos de construção e operação e com licenciamento ambiental.

Quando não observada a importância desta atividade são vários os danos decorrentes como contaminação do meio ambiente, acidentes de trabalho envolvendo profissionais da saúde, da limpeza pública e catadores, além da propagação de doenças para a população em geral, por contato direto ou indireto, através de vetores.

Dentro do contexto descrito, a conscientização dos profissionais para o cuidado com a segregação dos resíduos gerados durante sua atuação no ambiente hospitalar e também relevante por proporcionar uma visão ampliada das questões ambientais da atualidade, por despertar interesse e estimular sua participação nos programas de qualidade ambiental das unidades de saúde. Além disso, o conhecimento dos custos associados ao uso de materiais e insumos, e ao seu tratamento após uso, pode diminuir a utilização descontrolada e inadequada de materiais hospitalares. A expectativa é que profissionais de todos os níveis e áreas de atuação, conscientes da importância da correta segregação dos resíduos gerados nos serviços de saúde, sejam mais atuantes no processo, encaminhando apenas para tratamento aqueles materiais que realmente necessitem ser tratados.

Diante do exposto, professores e alunos bolsistas da Área de Saúde Coletiva do Curso de Enfermagem da Universidade Federal do Paraná - UFPR têm desenvolvido um projeto denominado "A Educação e o Processo de Trabalho da Enfermagem”, em um hospitalescola no município de Curitiba, com o objetivo de avaliar o impacto do treinamento em serviço no manejo dos resíduos dos serviços de saúde.

Esta pesquisa teve como objetivos: descrever como os profissionais do hospital-escola em questão relatam a aplicação dos conhecimentos adquiridos no treinamento em serviço sobre a segregação de resíduos de serviços de saúde e sua importância para o desenvolvimento de atividades diárias e comparar a descrição realizada pelos profissionais do hospitalescola que foram treinados para a segregação com o relato de profissionais do mesmo serviço que não foram treinados para tal.

\section{DESCRIÇÃO DO MÉTODO}

O projeto atua no hospital-escola desde 2005 e, ao todo, já capacitou 212 pessoas entre alunos dos cursos de saúde que freqüentam o hospital, residentes e trabalhadores. Também foram produzidos banners orientadores para a segregação correta dos resíduos. Os setores que tiveram funcionários capacitados foram: UTI - Neonatal, Centro Obstétrico, Cirurgia Pediátrica, Clínica Médica Feminina, Clínica Médica Masculina, Pediatria, Nefrologia, Urologia, Ortopedia, Cirurgia do Aparelho Digestivo, Hemodinâmica, CTCV (Cirurgia Torácica - Cirurgia Vascular), UTI Cardíaca, Cirurgia Geral, Neurocirurgia, Ginecologia, Banco de Leite Humano, Alojamento Conjunto, Banco de Sangue, UTI adulto, Transplante Hepático, UTI pediátrica, Neurologia, Centro Cirúrgico, Triagem e Otorrinolaringologia/Oftalmologia, totalizando 26 setores.

Para a realização da pesquisa foram acompanhados profissionais de 15 setores do hospital selecionados previamente e em que pelo menos uma pessoa havia sido capacitada através do projeto. De cada setor participaram dois profissionais: um que havia participado da capacitação e um que não participou. Os dados foram coletados nos três períodos, manhã, tarde e noite, por um período de duas horas de observação estruturada feita pelo pesquisador, quando procurou-se identificar quais os materiais foram utilizados, a classe à qual o resíduo pertencia, e o destino final dado pelo usuário.

Durante a observação estruturada e análise da destinação considerou-se a classe de cada resíduo de acordo com a resolução da ANVISA ${ }^{(1)}$. São elas: Grupo A: Resíduos com a possível presença de agentes biológicos que, por suas características de maior virulência ou concentração, podem apresentar risco de infecção. Devem ser acondicionados em saco branco leitoso. Grupo B: Resíduos contendo substâncias químicas que podem apresentar risco à saúde pública ou ao meio ambiente, dependendo de suas características de inflamabilidade, corrosividade, reatividade e toxicidade. Devem ser acondicionados 
de forma a observar as exigências de compatibilidade química dos resíduos entre si. Grupo C: Quaisquer materiais resultantes de atividades humanas que contenham radionuclídeos em quantidades superiores aos limites de isenção especificados nas normas do Conselho Nacional de Energia Nuclear (CNEN) e para os quais a reutilização é imprópria ou não prevista. Devem ser acondicionados em recipientes de material rígido, forrados internamente com sacos plásticos resistentes e identificados. Grupo D: Resíduos que não apresentam risco biológico, químico ou radiológico à saúde ou ao meio ambiente, podendo ser equiparados aos resíduos domiciliares. Devem ser acondicionados de acordo com as orientações dos serviços locais de limpeza urbana, utilizando-se sacos impermeáveis contidos em recipientes e receber identificação. Grupo E: Materiais perfurocortantes ou escarificantes. Devem ser descartados separadamente, no local de sua geração, imediatamente após o uso ou necessidade de descarte, em recipientes, rígidos, resistentes à punctura, ruptura e vazamento, com tampa, devidamente identificados e sendo expressamente proibido o esvaziamento desses recipientes para o seu reaproveitamento.

Para o desenvolvimento do estudo foram consideradas as Diretrizes e Normas Regulamentadoras da Pesquisa envolvendo Seres Humanos, de acordo com a Resolução 196 do Ministério da Saúde ${ }^{(6)}$. Foi fornecido aos participantes um Termo de Consentimento Livre e Esclarecido, garantindo-se total sigilo sobre os dados coletados e, também, a opção de participar ou não da pesquisa, tendo o direito de se recusar ou de se excluír do estudo quando julgassem necessário.

\section{RESULTADOS}

Analisando os dados coletados com os 30 profissionais dos 15 setores do hospital sobre o manejo e segregação de resíduos de serviços de saúde (RSS), percebemos que para a destinação do resíduo produzido nas atividades não houve diferença entre os funcionários que realizaram o treinamento em serviço e aqueles que não participaram do treinamento. Dos 30 funcionários que participaram da pesquisa, 20 cometeram erros durante a segregação.

Os erros mais comuns durante os descartes dos resíduos envolveram os grupos $\mathrm{A}$ e $\mathrm{D}$, resíduos infectantes e comuns, respectivamente. Em sete dos 15 setores foi evidenciada como situação mais marcante a destinação do algodão e da gaze não infectados, utilizados para a desinfecção de materiais, no caso, termômetros e estetoscópios, no lixo infectante. A Associação Brasileira de Normas Técnicas (ABNT), em sua NBR 12.807/93, define resíduo infectante (pertencente à classe $\mathrm{A}$ ) como aquele gerado em serviço de saúde que, por suas características de maior virulência, infectividade e concentração de patógenos, apresenta risco potencial adicional à saúde pública, enquanto o resíduo sólido da classe D é aquele originado da vida diária das residências, constituído por restos de alimentos como cascas de frutas, verduras, legumes, produtos deteriorados, jornais e revistas, garrafas, embalagens em geral, papel higiênico, fraldas descartáveis e uma grande diversidade de outros itens, incluindo produtos que podem ser tóxicos. Entretanto, existe uma orientação da Comissão de Gerenciamento de Resíduos do hospital estudado, já que uma possível fiscalização poderia encontrar esse tipo de material dentre materiais orgânicos não contaminados e considerar que a segregação está acontecendo de forma incorreta.

As embalagens recicláveis, na sua maior parte, foram para as lixeiras de resíduos não recicláveis. Isso mostra que ainda não há uma preocupação com o valor do material reciclável neste ambiente. Estas inadequações na destinação dos resíduos nos mostram que a geração de maior volume de material nos resíduos das Classes A e D poderão causar impactos no meio ambiente, com conseqüências posteriores para a sociedade.

Para o meio ambiente, o impacto da destinação incorreta causa degradação em decorrência do depósito de maior volume de resíduos que, neste caso, poderiam ser reaproveitados. Para a sociedade, de imediato, pode-se destacar como impacto o uso acelerado do espaço reservado para a destinação final do lixo, o que esgotaria este ambiente e exigiria uma outra área para tal finalidade. Outra questão seria $o$ fato de que, ao destinar corretamente o lixo, os próprios trabalhadores envolvidos no manejo dos resíduos poderiam se beneficiar da venda de resíduos recicláveis, ou mesmo a instituição produtora do lixo.

Entre as maiores dificuldades relatadas pelos sujeitos da pesquisa, durante a segregação de resíduos, estava a falta de local correto para descarte, de acordo com a classificação adotada no próprio local em que os resíduos são gerados. Este fato leva a um maior deslocamento dos trabalhadores durante a atividade, 
o que os estimula a destinar incorretamente o resíduo produzido devido à atividade que estão desenvolvendo. A organização do espaço utilizado pela equipe de enfermagem tem se constituído em um problema para a segregação de resíduos. Neste caso, percebe-se que o espaço é inadequado para a destinação, já que as lixeiras específicas se encontram centralizadas no posto de enfermagem. Essa observação reforça a fala do profissional que afirma que deverá se deslocar demais para desprezar o lixo produzido, interrompendo a atividade pela qual está responsável, e isso poderia até comprometer a assistência prestada.

Cabe ressaltar que tal organização para a destinação dos resíduos se deve ao fato de que as enfermarias não apresentam estrutura suficiente, com espaços específicos para a instalação de lixeiras para cada classe de resíduo.

Outro agravante para o problema em discussão é o descarte incorreto dos rejeitos pelos usuários internados e seus familiares visitantes, que descartam os resíduos não-infectantes, inclusive os recicláveis, inadequadamente, também pela falta das lixeiras específicas nos espaços por onde circulam. Além do grande número de pacientes e acompanhantes, em um Hospital Universitário existe intensa circulação de estudantes, com significativa rotatividade dos alunos dos vários cursos em todas as áreas do hospital. Isto reforça a necessidade de um treinamento anterior ao início das atividades de ensino-pesquisa, para que os estudantes estejam inteirados da importância da segregação dos resíduos. É preciso dar atenção especial à relação entre o ensino e o processo de trabalho em saúde, quando se pensa em mudança. As críticas feitas às instituições de ensino têm, com freqüência, denunciando a falta de sintonia destas com a realidade ${ }^{(7)}$.

A defasagem no treinamento do quadro de funcionários das unidades foi apontada como fator de relevância na ineficácia observada na destinação do resíduo. Em todos os setores encontramos, pelo menos em um turno, funcionários que não haviam sido treinados. Neste caso, cabe esclarecer que a capacitação foi ministrada de maneira sucinta, não abordando todos os diversos materiais a serem segregados que são produzidos no ambiente hospitalar e tratando apenas dos mais utilizados naquele local. Houve relatos de profissionais e chefes de unidades reforçando que é necessária uma capacitação contínua, que possibilitaria uma discussão mais aprofundada sobre os temas e estimularia um processo de reflexão sobre as realidades bastante diversas que encontramos nestes ambientes, bem como as suas conseqüências para o meio ambiente.

\section{CONSIDERAÇÕES FINAIS}

As novas legislações trazem avanços significativos com relação ao destino dos resíduos gerados pelos serviços de saúde. Por outro lado, são visíveis as dificuldades enfrentadas pelos serviços e profissionais para a adequação às normas vigentes.

O correto manejo dos RSS, em qual, apresenta como obstáculos a falta de estrutura para a separação dos resíduos no momento e local de sua geração. Esta situação dificulta a destinação dos resíduos para o local correto, de acordo com as características físicas, químicas, biológicas, e ainda de adequar conforme o seu estado físico e riscos envolvidos. Em um Hospital Universitário o comprometimento da destinação do resíduo na instituição, conforme observado, tem como agravante o grande número de profissionais, estudantes, pacientes e familiares visitantes, envolvidos no processo de segregação dos resíduos.

Para o enfrentamento das questões aqui apontadas, acredita-se que a capacitação da pessoas que circulam nas instalações dos serviços de saúde é fundamental. Ela deveria ser contínua e, de preferência, anteceder o início das atividades acadêmicas e de serviço, para que o impacto da destinação dos resíduos seja menor, tanto para a própria instituição como para o meio ambiente. Da mesma forma, o preparo do espaço físico para todas as etapas de segregação é uma exigência para o enfrentamento deste problema, cada vez mais presente na rotina dos serviços de saúde.

\section{REFERÊNCIAS}

1. Ministério da Saúde (BR). Agência Nacional de Vigilância Sanitária. Resolução da Diretoria Colegiada $\mathrm{n}^{\circ} 306$ de 7 de dezembro de 2004. Dispõe sobre o tratamento e a disposição final dos resíduos de serviços de saúde. Diário Oficial da União. 2004; 10 Dez.

2. Conselho Nacional do Meio Ambiente. Resolução n. 358 de 29 de abril de 2004. Dispõe sobre o tratamento e a disposição final dos resíduos dos serviços de saúde e dá outras providências. Diário Oficial da União 2005; 4 Mai.

3. Sisino CLS, Moreira JC. Ecoeficiência: um instrumento para a redução da geração de resíduos e desperdícios 
em estabelecimentos de saúde. Cad Saúde Publ. 2005;21(6):1893-1900.

4. Garcia LP, Zanetti - Ramos, BG. Gerenciamento dos resíduos de serviços de saúde: uma questão de biossegurança. Cad Saúde Publ. 2004;20(3):744-52.

5. Oliniski SR, Lacerda MR. As diferentes faces do ambiente de trabalho em saúde. Cogitare Enferm. 2004;9(2):43-52.

6. Ministério da Saúde (BR). Conselho Nacional de Saúde. Diretrizes e normas regulamentadoras de pesquisa em seres humanos. Resolução n ${ }^{\circ} 196$, de 10 de outubro de 1996. Brasília; 1996.

7. Gisi ML, Meier MJ, Muntsch SMA, Hamdar F. A relação ensino-serviço: estratégia de aproximação da formação acadêmica com o processo de trabalho em saúde. Cogitare Enferm. 1998;3(1):50-6. 